\title{
A highly efficient chemoenzymatic approach toward glycoprotein synthesis
}

\author{
Bing Li, Haijing Song, Steven Hauser, and Lai-Xi Wang* \\ Institute of Human Virology, University of Maryland Biotechnology Institute \\ University of Maryland, Baltimore, MD, 21201
}

\section{Supporting Information}

Experimental Section.......................................................S2-S6

References...............................................................................S6

${ }^{1} \mathrm{H}$ and ${ }^{13} \mathrm{C}$ NMR Spectra of key compounds................................S7-S13

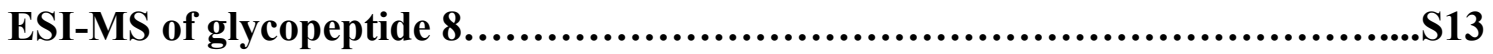




\section{Materials and methods}

All chemicals, biochemicals and reagents were reagent grade. TLC was performed on glass plates coated with silica gel 60 F254 (E. Merck). Flash column chromatography was performed on silica gel 60 (EM Science, 230-400 mesh). ${ }^{1} \mathrm{H}$ and ${ }^{13} \mathrm{C}$ NMR spectra were recorded on Inova 500 NMR machine. The chemical shifts were assigned in ppm. The ESI-MS spectra were measured on a micromass ZQ-4000 single quadruple mass spectrometer.

\section{Reverse-phase HPLC}

Analytical HPLC was carried out with a Waters HPLC instrument on a Waters C18 column $(3.9 \times 150 \mathrm{~mm})$ at $40{ }^{\circ} \mathrm{C}$. The column was eluted with a linear gradient of 0 $90 \% \mathrm{MeCN}$ containing $0.1 \%$ TFA at a flow rate of $1 \mathrm{~mL} / \mathrm{min}$ over 25 minutes. Peptides and glycopeptides were detected at double wavelengths (214 and $280 \mathrm{~nm}$ ). Preparative HPLC was performed with a Waters HPLC instrument of a Waters C18 column (Symmetry 300, 19x300 mm). The column was eluted with a suitable gradient of MeCN containing $0.1 \% \mathrm{TFA}$ at $12 \mathrm{~mL} / \mathrm{min}$.

\section{Synthesis of the oligosaccharide oxazolines}

\section{Ethyl 2,3,4,6-tetra-O-acetyl- $\beta$-D-galactopyranosyl-(1 $\rightarrow 4)-3-O-a c e t y l-2,6-d i-O-$ benzoyl-1-thio- $\alpha$-D-mannopyranoside (1)}

A suspension of 2,3,4,6-tetra-O-acetyl- $\alpha$-D-galactopyranosyl imidate ${ }^{[1]}$ ( $770 \mathrm{mg}$, $1.56 \mathrm{mmol}$ ) and ethyl 2,6-di-O-benzoyl-1-thio- $\alpha$-D-mannopyranoside ${ }^{[2]}$ (560 mg, 1.29 mmol) containing activated 4 Á molecular sieves (500 mg) in dry $\mathrm{CH}_{2} \mathrm{Cl}_{2}$ (30 ml) was stirred under an atmosphere of argon at r.t. for $30 \mathrm{~min}$. After cooling to $-78^{\circ} \mathrm{C}, 0.1 \mathrm{M}$ TMSOTf in $\mathrm{CH}_{2} \mathrm{Cl}_{2}(0.2 \mathrm{ml}, 0.02 \mathrm{mmol})$ was added and the mixture was stirred at $-78^{\circ} \mathrm{C}$ to r.t. overnight. The reaction was quenched with $\mathrm{Et}_{3} \mathrm{~N}$ and the mixture was filtered. The filtrate was washed with $\mathrm{NaHCO}_{3}$ solution and brine, dried over $\mathrm{Na}_{2} \mathrm{SO}_{4}$, and filtered. The filtrate was concentrated in vacuo and the residue was subjected to flash silica gel column chromatography (5:1 Hexanes: EtOAc) to afford two disaccharides (840 mg, $85 \%)$ as white foams. To this mixture, $\mathrm{Py}(10 \mathrm{ml})$ and $\mathrm{Ac}_{2} \mathrm{O}(5 \mathrm{ml})$ were added and the reaction was stirred at r.t. overnight. The mixture was diluted with $\mathrm{CH}_{2} \mathrm{Cl}_{2}(50 \mathrm{ml})$ and washed with saturated $\mathrm{NaHCO}_{3}, \mathrm{HCl}(1 \mathrm{~N})$ and $\mathrm{H}_{2} \mathrm{O}$, dried over $\mathrm{Na}_{2} \mathrm{SO}_{4}$, and filtered. The filtrate was concentrated in vacuo and the residue was subject to flash silica gel column chromatography (3:1 Hexanes: EtOAc) to give the $\beta$-1,4-linked disaccharide 1 (302 mg,

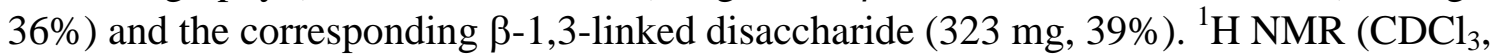
$500 \mathrm{MHz}$ ) of compound 1: $\delta 8.13(\mathrm{~d}, 2 \mathrm{H}, J=7.5 \mathrm{~Hz}, \mathrm{Ar}-), 8.06$ (d, 2H, $J=8.0 \mathrm{~Hz}, \mathrm{Ar}-)$, 7.68-7.66 (m, 1H, Ar-), 7.63-7.61 (m, 1H, Ar-), 7.53-7.50 (m, 1H, Ar-), 7.42-7.39 (m, $1 \mathrm{H}, \mathrm{Ar}-)$, 5.68 (s, $1 \mathrm{H}, \mathrm{H}-2^{\mathrm{Man}}$ ), 5.46 (dd, $\left.1 \mathrm{H}, J=10 \mathrm{~Hz}, \mathrm{H}-3^{\mathrm{Man}}\right), 5.41$ (s, $1 \mathrm{H}, \mathrm{H}-1^{\text {Man }}$ ), 5.37 (s, $1 \mathrm{H}, \mathrm{H}-4^{\mathrm{Gal}}$ ), 5.23 (dd, $1 \mathrm{H}, J=8.0 \mathrm{~Hz}, 10.0 \mathrm{~Hz}, \mathrm{H}-2^{\mathrm{Gal}}$ ), 4.99 (d, $1 \mathrm{H}, J=10.5 \mathrm{~Hz}$, H-3 ${ }^{\mathrm{Gal}}$ ), 4.79 (d, $1 \mathrm{H}, J=11.5 \mathrm{~Hz}, \mathrm{H}-6^{\text {Man }}$ a), 4.70 (d, $1 \mathrm{H}, J=7.5 \mathrm{~Hz}, \mathrm{H}-6^{\text {Man }}$ b), 4.574.55 (m, 2H, H-5 $\left.{ }^{\text {Man }}, \mathrm{H}-1^{\mathrm{Gal}}\right), 4.34$ (t, $\left.1 \mathrm{H}, J=9.5 \mathrm{~Hz}, \mathrm{H}-4^{\mathrm{Man}}\right)$, 4.19-4.13 (m, 1H, H-6 ${ }^{\mathrm{Gal}}$ a), 4.09-4.05 (m, $\left.1 \mathrm{H}, \mathrm{H}-6{ }^{\mathrm{Gal}} \mathrm{b}\right), 3.90-3.87$ (m, $\left.1 \mathrm{H}, \mathrm{H}-5^{\mathrm{Gal}}\right), 2.74-2.69$ (m, $2 \mathrm{H}, \mathrm{SC}_{2} \mathrm{CH}_{3}-$ 
), 2.20 (s, 3H, $\mathrm{CH}_{3} \mathrm{CO}_{2^{-}}$), 2.07 (s, 6H, $2 \mathrm{CH}_{3} \mathrm{CO}_{2^{-}}$), 2.01 (s, 3H, $\mathrm{CH}_{3} \mathrm{CO}_{2^{-}}$), 1.94 (s, 3H, $\left.\left.\mathrm{CH}_{3} \mathrm{CO}_{2-}\right), 1.69 \mathrm{~s}, 1 \mathrm{H}, \mathrm{SCH}_{2} \mathrm{CH}_{3}-\right) ;{ }^{13} \mathrm{C} \mathrm{NMR}\left(\mathrm{CDCl}_{3}, 125 \mathrm{MHz}\right)$ of compound 1: $\delta$ 170.41, 170.25, 170.15, 169.48, 169.31, 165.98, 165.26, 133.49, 133.38, 129.89, 129.80, 129.63, 129.55, 128.68, 128.60, 101.06, 82.23, 74.23, 71.77, 71.05, 70.55, 70.29, 69.74, 69.29, 66.76, 62.77, 61.90, 25.53, 20.84, 20.71, 20.69, 20.56, 20.41, 14.83.

${ }^{1} \mathrm{H} \mathrm{NMR}\left(\mathrm{CDCl}_{3}, 500 \mathrm{MHz}\right)$ of the corresponding $\beta$-1,3-linked disaccharide: $\delta 8.12(\mathrm{~d}$, 2H, $J=7.0 \mathrm{~Hz}, \mathrm{Ar}-$ ), 8.07 (d, 2H, $J=7.0 \mathrm{~Hz}, \mathrm{Ar}-$ ), 7.63-7.60 (m, 2H, Ar-), 7.47-7.44 (m, 4H, Ar-), 5.61 (t, 1H, J = 9.0 Hz, H-4), 5.50 (s, 1H, H-2), 5.45 (s, 1H, H-4'), 5.36 (s, 1H, H-1), 5.09-5.05 (m, 1H, H-2'), 4.97-4.95 (m, 1H, H-3’), 4.59-4.56 (m, 2H, H-6a, H-1), 4.51-4.49 (m, 1H), 4.44-4.42 (m, 1H), 4.31-4.29 (m, 1H), 4.20-4.16 (m, 1H), 4.13-4.09 (m, 1H), 3.94-3.92 (m, 1H), 2.75-2.70 (m, 2H, $\left.\mathrm{SCH}_{2} \mathrm{CH}_{3}-\right), 2.17$ (s, 3H, $\mathrm{CH}_{3} \mathrm{CO}_{2^{-}}$), 2.16 (s, $3 \mathrm{H}, \mathrm{C}_{3} \mathrm{CO}_{2^{-}}$), 2.12 (s, $3 \mathrm{H}, \mathrm{C}_{3} \mathrm{CO}_{2^{-}}$), 2.09 (s, $3 \mathrm{H}, \mathrm{CH}_{3} \mathrm{CO}_{2^{-}}$), 1.96 (s, 3H, $\underline{\mathrm{C}}_{3} \mathrm{CO}_{2^{-}}$), 1.65 (s, 3H, $\mathrm{CH}_{3} \mathrm{CO}_{2}^{-}$).

Benzyl 2,3,4,6-tetra-O-acetyl- $\beta$-D-galactopyranosyl-(1 $\rightarrow 4)-3-O$-acetyl-2,6-di-Obenzoyl- $\alpha$-D-mannopyranosyl- $(1 \rightarrow 3)$-[ $2,3,4,6$-tetra-O-acetyl- $\beta$-D-galactopyranosyl$(1 \rightarrow 4)-3-O$-acetyl-2,6-di-O-benzoyl- $\alpha$-D-mannopyranosyl-( $\rightarrow 6)]-2-O-b e n z o y l-4-O-$ benzyl- $\beta$-D-mannopyranosyl-( $1 \rightarrow 4)-3,6$-di-O-benzyl-2-deoxy-2-phthalamido- $\beta$-Dglucopyranoside (3)

A suspension of donor $\mathbf{1}$ (180 $\mathrm{mg}, 0.22 \mathrm{mmol}$ ) and acceptor $\mathbf{2}^{[3]}$ (70 mg, 0.07 mmol) containing activated 4 Á molecular sieves $(200 \mathrm{mg})$ in dry $\mathrm{CH}_{2} \mathrm{Cl}_{2}(10 \mathrm{ml})$ was stirred under an atmosphere of argon at r.t. for $30 \mathrm{~min}$. After cooling to $-40^{\circ} \mathrm{C}$, NIS (53 $\mathrm{mg}, 0.24 \mathrm{mmol}$ ) and AgOTf (6 mg, $0.02 \mathrm{mmol}$ ) were added and the mixture was stirred at $-40^{\circ} \mathrm{C}$ to r.t. overnight. The reaction was quenched with $\mathrm{Et}_{3} \mathrm{~N}$ and the mixture was filtered. The filtrate was washed with $\mathrm{Na}_{2} \mathrm{~S}_{2} \mathrm{O}_{3}$ solution and brine, dried over $\mathrm{Na}_{2} \mathrm{SO}_{4}$, and filtered. The filtrate was concentrated in vacuo and the residue was subject to flash silica gel column chromatography (2:1 Hexanes: EtOAc) to afford 3 (172 mg, 95\%) as a white foam. ${ }^{1} \mathrm{H} \mathrm{NMR}\left(\mathrm{CDCl}_{3}, 500 \mathrm{MHz}\right): \delta 8.23-7.01$ (m, 49H, Ar), 6.46 (s, 1H), 6.31-6.28 (m, 4H), 5.71 (s, 1H), 5.67 (s, 1H), 5.61 (s, 1H), 5.58 (d, 1H, $J=9.5 \mathrm{~Hz}), 5.43$ (d, 1H, $J=9.0$ $\mathrm{Hz}$ ), 5.38 (s, 1H), 5.34(s, 1H), 5.22-5.16 (m, 4H), 5.06-4.95(m, 5H), 4.86-4.62 (m, 6H), 4.57-4.55 (m, 2H), 4.50-4.35 (m, 4H), 4.30-4.24 (m, 2H), 4.18-4.03 (m, 5H), 4.00-3.90 (m, 5H), 3.83-3.81 (m, 2H), 3.69 (d, 1H, $J=8.5 \mathrm{~Hz}$ ), 3.49 (d, 1H, $J=9.5 \mathrm{~Hz}$ ), 3.25 (d, $1 \mathrm{H}, J=9.0 \mathrm{~Hz}$ ), 2.20 (s, 3H, $\mathrm{C}_{3} \mathrm{CO}_{2^{-}}$), 2.17 (s, 3H, $\underline{\mathrm{C}}_{3} \mathrm{CO}_{2^{-}}$), 2.10 (s, 3H, $\underline{\mathrm{H}}_{3} \mathrm{CO}_{2^{-}}$),

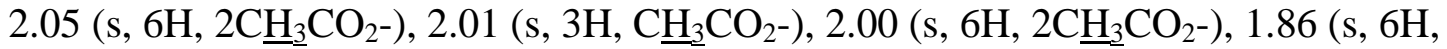

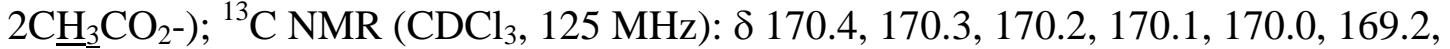
166.3, 166.0, 164.9, 164.6, 138.3, 138.0, 137.9, 137.2, 133.4, 133.2, 132.9, 130.2, 130.0, 129.8, 129.7, 129.6, 129.3, 129.0, 128.8, 128.6, 128.5, 128.3, 128.2, 128.1, 128.0, 127.9, 127.6, 127.5, 127.3, 126.5, 123.0, 101.1, 100.9, 100.3, 99.3, 98.0, 97.2, 81.8, 79.4, 76.9, 75.7, 75.3, 75.1, 74.4, 74.3, 74.1, 74.0, 73.9, 73.5, 73.3, 71.5, 71.2, 71.1, 70.6, 70.5, 70.4, 70.3, 70.1, 70.0, 69.7, 69.6, 69.3, 69.0, 68.9, 68.6, 66.9, 66.8, 65.3, 62.7, 62.4, 61.2, 61.1, 55.6, 29.7, 20.8, 20.7, 20.6, 20.3.

2,3,4,6-Tetra-O-acetyl- $\beta$-D-galactopyranosyl-( $1 \rightarrow 4)$-2,3,6-tri-O-acetyl- $\alpha$-Dmannopyranosyl-( $1 \rightarrow 3)$-[ $2,3,4,6$-tetra-O-acetyl- $\beta$-D-galactopyranosyl-(1 $\rightarrow 4)$-2,3,6tri-O-acetyl- $\alpha$-D-mannopyranosyl-(1 $\rightarrow 6)]-2,4-d i-O$-acetyl- $\beta$-D-mannopyranosyl$(1 \rightarrow 4)$-2-acetamido-1,3,6-tri-O-acetyl-2-deoxy- $\alpha, \beta$-D-glucopyranoside (4) 
A mixture of 3 (172 mg, $0.30 \mathrm{mmol})$ and $\mathrm{NH}_{2} \mathrm{NH}_{2} \cdot \mathrm{H}_{2} \mathrm{O}(6 \mathrm{ml})$ in EtOH (30 ml) was heated at $90^{\circ} \mathrm{C}$ for $24 \mathrm{~h}$ and then concentrated in vacuo. To the oily residue were added pyridine $(5 \mathrm{ml})$ and $\mathrm{Ac}_{2} \mathrm{O}(5 \mathrm{ml})$. The mixture was stirred at r.t. overnight. The mixture was diluted with $\mathrm{CH}_{2} \mathrm{Cl}_{2}(50 \mathrm{ml})$ and washed with saturated $\mathrm{NaHCO}_{3}, \mathrm{HCl}(1 \mathrm{~N})$ and brine, dried over $\mathrm{Na}_{2} \mathrm{SO}_{4}$, and filtered. The filtrate was concentrated in vacuo and the residue was subject to flash silica gel column chromatography (1:3 Hexanes: EtOAc) to give the corresponding 2-acetamido 2-deoxy compound as a white foam (129 $\mathrm{mg}, 90 \%$ ). ${ }^{1} \mathrm{H} \mathrm{NMR}\left(\mathrm{CDCl}_{3}, 500 \mathrm{MHz}\right): \delta$ 7.37-7.32 (m, 20H, Ar), 6.01 (d, $\left.1 \mathrm{H}, J=5.0 \mathrm{~Hz}\right), 5.42-$ 5.27 (m, 7H), 5.22-5.15 (m, 2H), 5.04-5.00 (m, 3H), 4.90-4.83 (m, 3H), 4.78-4.69 (m, 3H), 4.67-4.61 (m, 2H), 4.57-4.52(m, 6H), 4.35 (d, 1H, $\left.J=11.5 \mathrm{~Hz}, \mathrm{PhC}_{2_{2}}\right), 4.24$ (d, $1 \mathrm{H}, J=12 \mathrm{~Hz}, \mathrm{PhCH}_{2}-$ ), 4.19-4.03 (m, 7H), 3.96-3.89 (m, 6H), 3.81-3.78 (m, 3H), 3.713.60 (m, 5H), 3.23 (s, $1 \mathrm{H}), 2.20$ (s, 3H, $\left.\mathrm{CH}_{3} \mathrm{CO}_{2}-\right), 2.19$ (s, 3H, $\underline{\mathrm{C}}_{3} \mathrm{CO}_{2}$ ), 2.18 (s, 3H, $\mathrm{C}_{3} \mathrm{CO}_{2^{-}}$), 2.16 (s, $\left.9 \mathrm{H}, 3 \mathrm{CH}_{3} \mathrm{CO}_{2^{-}}\right), 2.12$ (s, 3H, $\left.\underline{\mathrm{CH}}_{3} \mathrm{CO}_{2^{-}}\right), 2.11$ (s, 3H, $\mathrm{CH}_{3} \mathrm{CO}_{2^{-}}$), 2.08 (s, $9 \mathrm{H}, 3 \mathrm{C}_{3} \mathrm{CO}_{2-}$ ), 2.01 (s, $\left.12 \mathrm{H}, 4 \mathrm{C}_{3} \mathrm{CO}_{2^{-}}\right), 1.85$ (s, 3H, $\left.\mathrm{C}_{3} \mathrm{CO}_{2^{-}}\right) ;{ }^{13} \mathrm{C} \mathrm{NMR} \mathrm{CDCl}_{3}$, $125 \mathrm{MHz}): \delta 171.7,170.8,170.7,170.5,170.4,170.3,170.2$, 170.1, 169.6, 169.5, 169.4, 169.3, 138.7, 138.2, 137.8, 137.5, 101.4, 100.9, 99.8, 97.6, 97.3, 80.3, 78.2, 76.1, 75.3, 74.7, 74.4, 74.3, 73.8, 73.4, 73.0, 71.1, 70.5, 70.4, 70.3, 69.7, 69.6, 69.5, 69.3, 69.2, 69.1, 68.9, 68.7, 66.8, 66.6, 66.4, 62.4, 62.2, 60.8, 60.4, 54.6, 29.7, 24.9, 23.3, 21.0, 20.9, 20.8, 20.7, 20.6, 14.2.

A mixture of the 2-acetamido-2-deoxy compound (129 mg, $64 \mu \mathrm{mol}$ ) and 10\% $\mathrm{Pd} / \mathrm{C}(50 \mathrm{mg})$ in $\mathrm{MeOH}(10 \mathrm{ml})$ was stirred under $\mathrm{H}_{2}$ atmosphere for $12 \mathrm{~h}$, and then filtered through Celite. The filtrate was concentrated in vacuo. To the residue were added pyridine $(5 \mathrm{ml})$ and $\mathrm{Ac}_{2} \mathrm{O}(5 \mathrm{ml})$ and the mixture was stirred at r.t. overnight. The mixture was diluted with $\mathrm{CH}_{2} \mathrm{Cl}_{2}$ and washed with saturated $\mathrm{NaHCO}_{3}, \mathrm{HCl}(1 \mathrm{~N})$, and brine, dried over $\mathrm{Na}_{2} \mathrm{SO}_{4}$ and filtered. The filtrate was concentrated in vacuo and the residue was subjected to flash silica gel column chromatography (1:2 Hexanes: EtOAc) to give 4 (92 mg, 78\%) as a white foam. ${ }^{1} \mathrm{H} \mathrm{NMR}\left(\mathrm{CDCl}_{3}, 500 \mathrm{MHz}\right): \delta 6.50(\mathrm{~d}, 1 \mathrm{H}, J=7.5 \mathrm{~Hz}), 6.25$ (d, 0.5H, $J=9.5 \mathrm{~Hz}), 6.17(\mathrm{~m}, 0.5 \mathrm{H}), 6.09(\mathrm{~s}, 1 \mathrm{H}), 6.01-5.99(\mathrm{~m}, 1 \mathrm{H}), 5.78-5.75(\mathrm{~m}, 1 \mathrm{H})$, $5.70(\mathrm{~d}, 0.5 \mathrm{H}, J=8.5 \mathrm{~Hz}), 5.55-5.54(\mathrm{~m}, 1 \mathrm{H}), 5.40-5.24(\mathrm{~m}, 4 \mathrm{H}), 5.24$ (s, 1H), 5.20-5.03 (m, 3H), 5.02-4.93 (m, 6H), $4.85(\mathrm{~s}, 0.5 \mathrm{H}), 4.79(\mathrm{~s}, 1 \mathrm{H}), 4.72-4.69(\mathrm{~m}, 1 \mathrm{H}), 4.59-4.46$ (m, $3 \mathrm{H})$, 4.36-4.28 (m, 2H), 4.21-4.11 (m, 4H), 4.07-3.93 (m, 9H), 3.86-3.82 (m, 1H), 3.81-

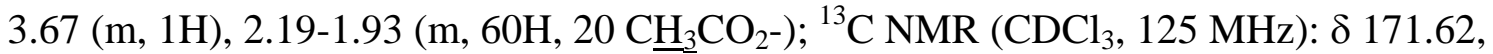
171.16, 171.11, 170.80, 170.64, 170.52, 170.48, 170.40, 170.31, 170.22, 170.15, 170.01, 169.76, 169.55, 169.49, 169.29, 169.13, 169.03, 107.30, 101.21, 100.98, 97.59, 97.14, 96.88, 92.35, 90.66, 75.19, 74.13, 73.46, 72.35, 71.05, 70.00, 70.39, 70.34, 70.17, 69.65, 69.25, 69.20, 69.10, 69.02, 68.61, 68.26, 67.19, 66.66, 66.54, 62.12, 62.02, 61.90, 60.80, 60.39, 51.82, 50.90, 47.09, 37.47, 29.68, 24.92, 24.62, 22.99, 22.83, 21.35, 21.05, 20.99, 20.94, 20.87, 20.79, 20.73, 20.64, 20.57.

\section{2,3,4,6-Tetra-O-acetyl- $\beta$-D-galactopyranosyl-( $1 \rightarrow 4)$-2,3,6-tri-O-acetyl- $\alpha$-D- mannopyranosyl-( $1 \rightarrow 3)$-[ 2,3,4,6-tetra-O-acetyl- $\beta$-D-galactopyranosyl-( $1 \rightarrow 4)-2,3,6$ -

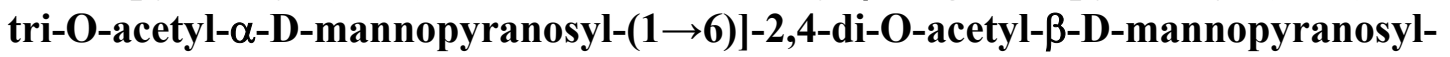 $(1 \rightarrow 4)-1,2-d i-d e o x y-\alpha-D$-glucopyrano-[2,1-d]-2-oxazoline (5)}

Compound 4 (47 mg, $26 \mu \mathrm{mol}$ ) was dissolved in anhydrous $\mathrm{ClCH}_{2} \mathrm{CH}_{2} \mathrm{Cl}(3 \mathrm{ml})$ in an oven-dried flask, under an atmosphere of argon. To the solution were added $\mathrm{TMSBr}$ (17 $\mu \mathrm{l}, 128 \mu \mathrm{mol}), \mathrm{BF}_{3} \mathrm{Et}_{2} \mathrm{O}(16 \mu \mathrm{l}, 128 \mu \mathrm{mol})$ and 2, 4, 6-collidine $(17 \mu \mathrm{l}, 128 \mu \mathrm{mol})$. 
The mixture was stirred at r.t. for $5 \mathrm{~h}$ when TLC indicated the completion of the reaction. The mixture was diluted with $\mathrm{CH}_{2} \mathrm{Cl}_{2}$ and washed with saturated $\mathrm{NaHCO}_{3}$ and brine. The organic layer was dried over $\mathrm{Na}_{2} \mathrm{SO}_{4}$ and filtered. The filtrate was concentrated in vacuo and the residue was purified by flash silica gel column chromatography (300:1 EtOAc: $\left.\mathrm{Et}_{3} \mathrm{~N}\right)$ to give the peracetated oxazoline derivative $5(27 \mathrm{mg}, 60 \%)$ as a white solid. ${ }^{1} \mathrm{H}$ NMR (CDCl $3,500 \mathrm{MHz}$ ): $\delta 5.97$ (d, $1 \mathrm{H}, J=7.5 \mathrm{~Hz}, \mathrm{H}-1), 5.66$ (s, $1 \mathrm{H}, \mathrm{H}-3$ ), 5.43-5.40 (m, 3H), 5.31-5.24 (m, 3H), 5.18-5.09 (m, 3H), 5.01-4.96 (m, 3H), 4.84 (s, 1H), 4.75 (s, $1 \mathrm{H})$, 4.61-4.49 (m, 4H), 4.28-4.15 (m, 7H), 4.10-4.05 (m, 3H), 3.99-3.87 (m, 7H), 3.743.67 (m, 3H), 3.58-3.56 (m, 1H), 3.46-3.45 (m, $1 \mathrm{H}), 2.24$ (s, 3H, $\left.\mathrm{CH}_{3} \mathrm{CO}_{2^{-}}\right), 2.21$ (s, $6 \mathrm{H}$, $2 \mathrm{CH}_{3} \mathrm{CO}_{2}$ ), 2.19 (s, $6 \mathrm{H}, 2 \mathrm{CH}_{3} \mathrm{CO}_{2^{-}}$), 2.18 (s, $6 \mathrm{H}, 2 \mathrm{CH}_{3} \mathrm{CO}_{2^{-}}$), 2.15 (s, $3 \mathrm{H}, \mathrm{C}_{3} \mathrm{CO}_{2}$ ), 2.14 (s, $3 \mathrm{H}, \mathrm{C}_{3} \mathrm{CO}_{2}-$ ), 2.13 (s, $3 \mathrm{H}, \mathrm{CH}_{3} \mathrm{CO}_{2}$ ), 2.10 (s, $15 \mathrm{H}, 5 \mathrm{C}_{3} \mathrm{CO}_{2}$ ), 2.06 (s, $3 \mathrm{H}$, $\mathrm{C}_{3} \mathrm{CO}_{2}$ ), 2.05 (s, 3H, $\mathrm{C}_{3} \mathrm{CO}_{2}$ ), 2.02 (s, 6H, $2 \mathrm{C}_{3} \mathrm{CO}_{2-}$ ); ${ }^{13} \mathrm{C} \mathrm{NMR}\left(\mathrm{CDCl}_{3}, 125\right.$ $\mathrm{MHz}): \delta$ 170.7, 170.6, 170.4, 170.3, 170.2, 170.0, 169.8, 169.1, 166.3, 101.2, 100.9, 99.6, 99.4, 99.1, 97.5, 74.3, 73.6, 72.9, 71.1, 70.4, 70.3, 70.1, 70.0, 69.8, 69.4, 69.1, 68.9, 68.0, 66.7, 64.2, 63.6, 62.5, 62.0, 60.8, 60.7, 60.4, 20.9, 20.7, 20.6, 20.5, 14.2, 13.7.

\section{$\beta$-D-Galactopyranosyl-(1 $\rightarrow 4)-\alpha-D$-mannopyranosyl-( $\rightarrow 3)-[\beta$-D-galactopyranosyl- $(1 \rightarrow 4)$ - $\alpha$-D-mannopyranosyl- $(1 \rightarrow 6)]-\beta$-D-mannopyranosyl-(1 $\rightarrow 4)-1,2$-di-deoxy- $\alpha$-D- glucopyrano-[2,1-d]-2-oxazoline (6)}

A solution of the peracetylated oxazoline 5 (27 mg, $15 \mu \mathrm{mol})$ in $\mathrm{MeOH}(2 \mathrm{ml})$ containing MeONa $(3.0 \mu \mathrm{mol})$ was stirred at r.t. overnight. The $\mathrm{MeOH}$ was evaporated and the residue was dissolved in water and lyophilized to give the oxazoline 6 (15 mg, quantitative) as a white solid. ${ }^{1} \mathrm{H}$ NMR ( $\left.\mathrm{D}_{2} \mathrm{O}, 500 \mathrm{MHz}\right): \delta 6.02(\mathrm{~d}, 1 \mathrm{H}, J=6.5 \mathrm{~Hz}, \mathrm{H}-1)$, 5.02 (s, $\left.1 \mathrm{H}, \mathrm{H}-1^{\alpha-\mathrm{Man}}\right), 4.88\left(\mathrm{~s}, 1 \mathrm{H}, \mathrm{H}-1^{\alpha-M a n}\right), 4.67$ (s, $\left.1 \mathrm{H}, \mathrm{H}-1^{\beta-M a n}\right), 4.37-4.34(\mathrm{~m}, 3 \mathrm{H}, \mathrm{H}-$ $\left.2^{\beta-M a n}, 2 \mathrm{H}-1^{\beta-G a l}\right), 4.07$ (s, 1H), 4.05 (s, 1H), 4.00 (s, 1H), 3.92 (s, 1H), 3.90-3.67 (m, 22H), 3.58-3.45 (m, 6H), 3.33-3.32 (m, $1 \mathrm{H}), 3.27$ (s, $1 \mathrm{H}), 1.99$ (s, $\left.3 \mathrm{H}, \mathrm{C}_{3}{ }^{-}\right) ;{ }^{13} \mathrm{C} \mathrm{NMR}$ $\left(\mathrm{D}_{2} \mathrm{O}, 125 \mathrm{MHz}\right): \delta 168.3,103.1,103.0,102.2,101.4,99.9,99.4,80.9,78.1$, 76.5, 75.4, 74.3, 72.6, 72.0, 71.3, 71.0, 70.9, 70.3, 69.6, 69.4, 69.1, 69.0, 68.6, 65.8, 65.1, 61.8, 61.1, 60.5, 60.3, 13.0 .

\section{Synthesis of glycopeptide (8)}

A mixture of the hexasaccharide oxazoline $6(0.69 \mathrm{mg}, 0.68 \mu \mathrm{mol})$ and the GlcNAc-tripeptide 7 (0.2 mg, $0.34 \mu \mathrm{mol})$ in a phosphate buffer (50 mM, pH 6.5, $100 \mu \mathrm{l}$ ) was incubated at $23^{\circ} \mathrm{C}$ with the enzyme Endo-A (200 mU). The reaction was monitored by analytical HPLC on a Waters Nova-Pak C18 column $(3.9 \times 150 \mathrm{~mm})$ at $40^{\circ} \mathrm{C}$ with a linear gradient (0-90\% MeCN containing 0.1\% TFA in $25 \mathrm{~min}$, flow rate $1 \mathrm{ml} / \mathrm{min}$ ). Within 30 min, the GlcNAc-tripeptide was completely converted to a new species that was eluted slightly earlier than the starting material. The product was purified by preparative HPLC on a Waters preparative column (Symmetry 300, 19x300 mm) to afford the glycopeptide $8(0.53 \mathrm{mg}, 98 \%) .{ }^{1} \mathrm{H}$ NMR $\left(\mathrm{D}_{2} \mathrm{O}, 500 \mathrm{MHz}\right): \delta 5.05(\mathrm{~s}, 1 \mathrm{H}, \mathrm{H}-$ $\left.1^{\alpha-M a n}\right), 4.99\left(\mathrm{~d}, 1 \mathrm{H}, J=8.5 \mathrm{~Hz}, \mathrm{H}-1^{\text {GlcNAc-1}}\right), 4.87\left(\mathrm{~s}, 1 \mathrm{H}, \mathrm{H}-1^{\alpha-M a n}\right), 4.67\left(\mathrm{~s}, 1 \mathrm{H}, \mathrm{H}-1^{\beta-M a n}\right)$, 4.56 (d, $1 \mathrm{H}, J=7.5 \mathrm{~Hz}, \mathrm{H}-1^{\text {GlcNAc-2}}$ ), $), 4.40$ (s, $\left.1 \mathrm{H}, \mathrm{H}-1^{\beta-G a l}\right), 4.38$ (s, $\left.1 \mathrm{H}, \mathrm{H}-1^{\beta-G a l}\right), 4.26$ (d, 1H, $J=4.4$ Hz, Thr-H ${ }_{\alpha}$ ), 4.21-4.19 (m, 3H), 4.08 (s, 1H), 3.99 (s, 1H), 3.94-3.50 (m, 38H), 2.78 (dd, $1 \mathrm{H}, J=5.8,16.0 \mathrm{~Hz}, \mathrm{Asn}_{-} \mathrm{H}_{\beta}$ ), 2.65 ( dd, $1 \mathrm{H}, J=16.0,7.8 \mathrm{~Hz}, \mathrm{Asn}-\mathrm{H}_{\beta}$ ), 2.01 (s, 3H, $\mathrm{NHCOC}_{\underline{H}_{3}}$ ), 1.95 (s, 3H, $\mathrm{NHCOC}_{\underline{H}_{3}}$ ), 1.89-1.85 (m, $1 \mathrm{H}$, Leu- $\mathrm{H}_{\beta}$ ), 1.15 (d, 
3H, $J=5.5 \mathrm{~Hz}$, Thr- $\mathrm{CH}_{3}-$ ), 0.87 ( d, 3H, $J=6.5 \mathrm{~Hz}$, Leu-C $\underline{H}_{3}^{-}$), 0.82 (t, 3H, $J=7.0 \mathrm{~Hz}$, Leu-C $\left.\underline{\mathrm{H}}_{3}{ }^{-}\right)$; ESI-MS: MS = 1604.5; Found, $1605.0(\mathrm{M}+\mathrm{H})^{+}, 1118.5(\mathrm{M}-2 \mathrm{Gal}-\mathrm{Man}+\mathrm{H})^{+}$, $956.5(\mathrm{M}-2 \mathrm{Gal}-2 \mathrm{Man}+\mathrm{H})^{+}, 721.8(\mathrm{M}-\mathrm{Gal}+2 \mathrm{H})^{2+}, 641.3(\mathrm{M}-2 \mathrm{Gal}+2 \mathrm{H})^{2+}, 559.9(\mathrm{M}-2 \mathrm{Gal}-$ $\mathrm{Man}+2 \mathrm{H})^{2+}, 478.8(\mathrm{M}-2 \mathrm{Gal}-2 \mathrm{Man}+2 \mathrm{H})^{2+}$.

\section{Preparation of GIcNAc-containing Ribonuclease B (9) - (GIcNAc-RB)}

The bovine Ribonuclease B obtained from Sigma was purified by boratephosphate cation exchange chromatography according to the reported procedure. ${ }^{[3\}}$ The purified Ribonuclease B (50 mg) was dissolved in a phosphate buffer (50 mM, PH 6.0, $2 \mathrm{ml})$. To the solution was added Endo-H (500 $\mathrm{mU})$ and the mixture was incubated at $37^{\circ} \mathrm{C}$ for $5 \mathrm{~h}$. The partially de-glycosylated product was purified by preparative HPLC to give the GlcNAc-RB (9) (36 mg, 80\%). ESI-MS: calculated MS = 13886; Found, 1737 $(\mathrm{M}+8 \mathrm{H})^{8+}, 1544(\mathrm{M}+9 \mathrm{H})^{9+}, 1390(\mathrm{M}+10 \mathrm{H})^{10+}, 1263(\mathrm{M}+11 \mathrm{H})^{11^{+}}, 1158(\mathrm{M}+$ $12 \mathrm{H})^{12+}, 1069(\mathrm{M}+13 \mathrm{H})^{13+}, 993(\mathrm{M}+14 \mathrm{H})^{14+}$.

\section{Synthesis of glycoprotein (10) - $\left(\mathrm{Gal}_{2} \mathrm{Man}_{3} \mathrm{GIcNAc}_{2}-\mathrm{RB}\right)$}

A mixture of the hexasaccharide oxazoline $6(0.57 \mathrm{mg}, 0.56 \mu \mathrm{mol})$ and the GlcNAc-RNB protein (3.9 mg, $0.28 \mu \mathrm{mol}$ ) in a phosphate buffer (50 mM, pH 6.5, 150 $\mu \mathrm{l})$ was incubated at $23^{\circ} \mathrm{C}$ with the enzyme Endo-A (200 mU). The reaction was monitored by analytical HPLC on a Waters Nova-Pak C18 column $(3.9 \times 150 \mathrm{~mm})$ at $40^{\circ} \mathrm{C}$ with a linear gradient (0-90\% MeCN containing $0.1 \%$ TFA in 25 min, flow rate 1 $\mathrm{ml} / \mathrm{min}$ ). Within $2 \mathrm{~h}$, the GlcNAc-RB protein was completely converted to a new species that was eluted slightly earlier than GlcNAc-RB. The product was purified by preparative HPLC on a Waters preparative column (Symmetry 300, 19x300 mm) to afford the glycoprotein 10 (4.02 mg, 96\%). ESI-MS: calculated MS =14900; Found, 1863.4 (M + $8 \mathrm{H})^{8+}, 1656.4(\mathrm{M}+9 \mathrm{H})^{9+}, 1490.9(\mathrm{M}+10 \mathrm{H})^{10+}, 1355.5(\mathrm{M}+11 \mathrm{H})^{11^{+}}, 1242.6(\mathrm{M}+$ $12 \mathrm{H})^{12+}, 1147.1(\mathrm{M}+13 \mathrm{H})^{13+}, 1065.2(\mathrm{M}+14 \mathrm{H})^{14+}$.

\section{Synthesis of glycoprotein (12) - $\left(\mathrm{Man}_{3} \mathrm{GlcNAc}_{2}-\mathrm{RB}\right)$}

The Endo-A catalyzed transglycosylation between the tetra-saccharide oxazoline $\mathbf{1 1}^{[4]}$ and the GlcNAc-RB 9 protein was performed in the same way as the synthesis of glycoprotein 10 to afford the glycoprotein 12 in 82\% isolated yield. ESI-MS: calculated MS = 14575; Found, $1823(\mathrm{M}+8 \mathrm{H})^{8+}, 1620(\mathrm{M}+9 \mathrm{H})^{9+}, 1459(\mathrm{M}+10 \mathrm{H})^{10+}, 1326(\mathrm{M}+$ $11 \mathrm{H})^{11+}, 1216(\mathrm{M}+12 \mathrm{H})^{12+}, 1122(\mathrm{M}+13 \mathrm{H})^{13+}, 1042(\mathrm{M}+14 \mathrm{H})^{14+}$.

\section{References}

[1] Nakahara, Y.; Shibayama, S.; Ogawa, T. Carbohydr. Res., 1996, 280, 67-84.

[2] Oscarson, S.; Svahnberg, P. Carbohydr. Res., 1998, 309, 207-212.

[3] Witte, K.; Sears, P.; Martin, R.; Wong, C. H. J. Am. Chem. Soc. 1997, 119, 21142118.

[4] Li, B.; Zeng, Y.; Hauser, S.; Song, H.; Wang, L. X. J. Am. Chem. Soc., 2005, 127, 9692-9693. 

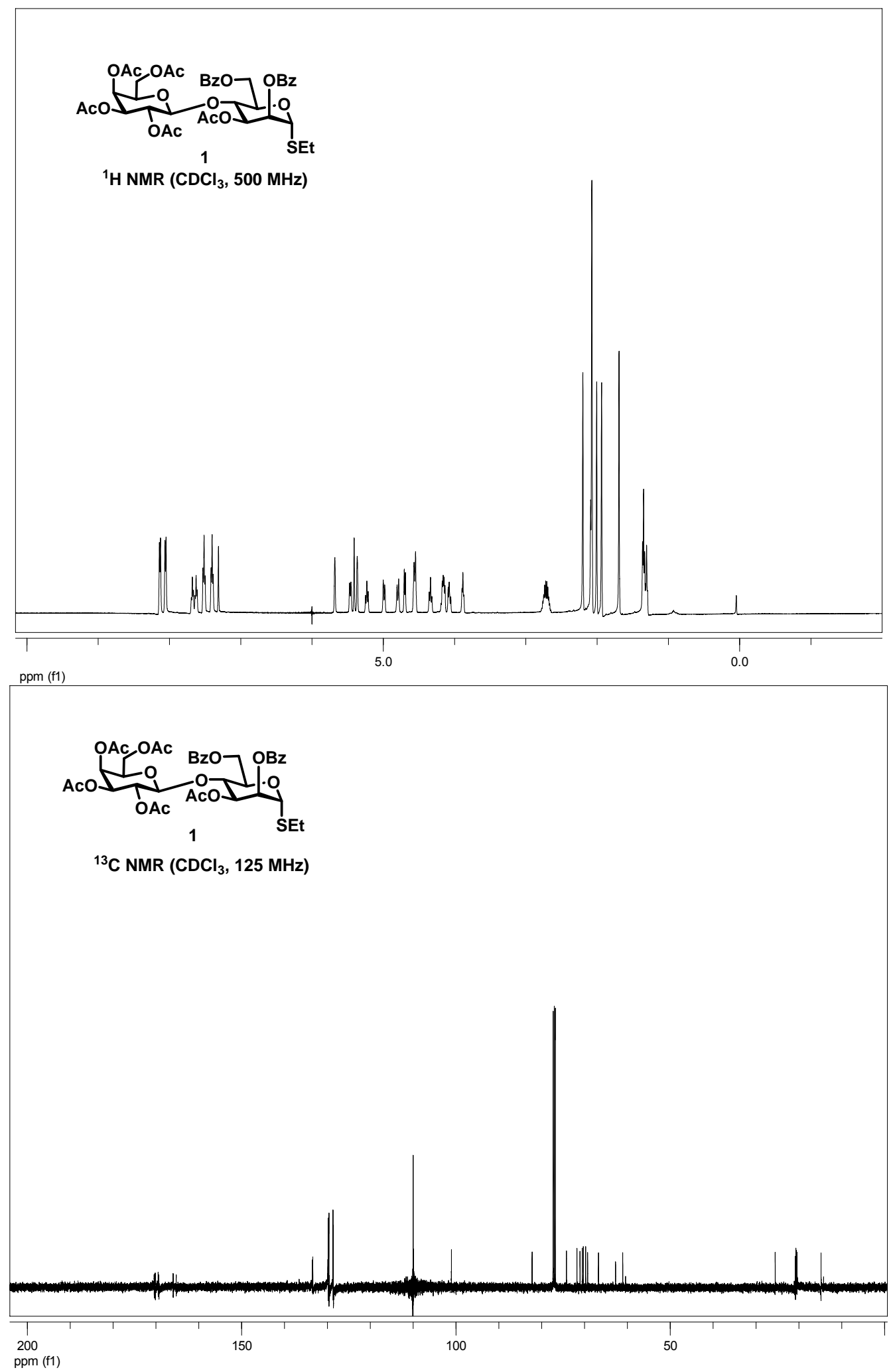

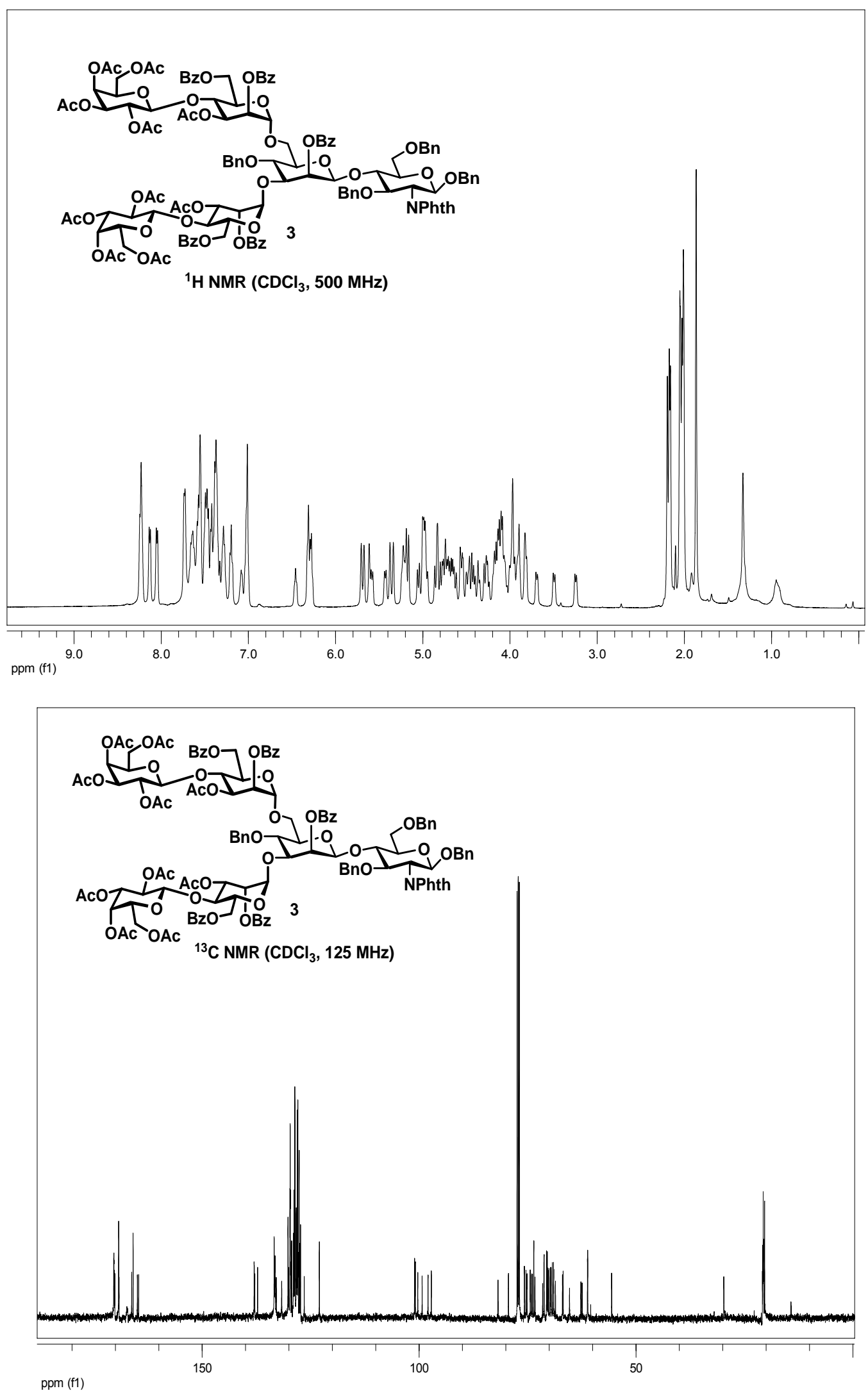

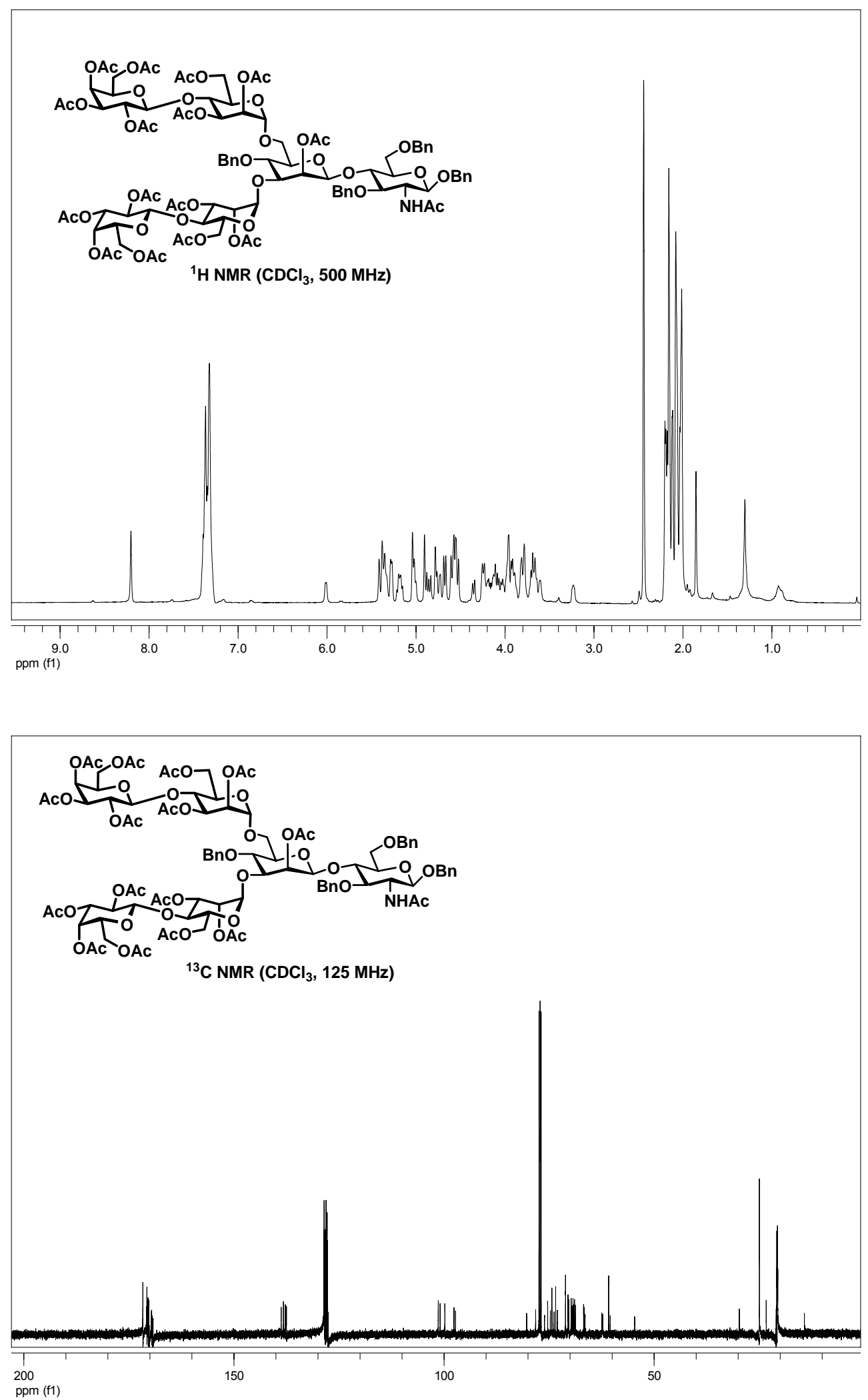


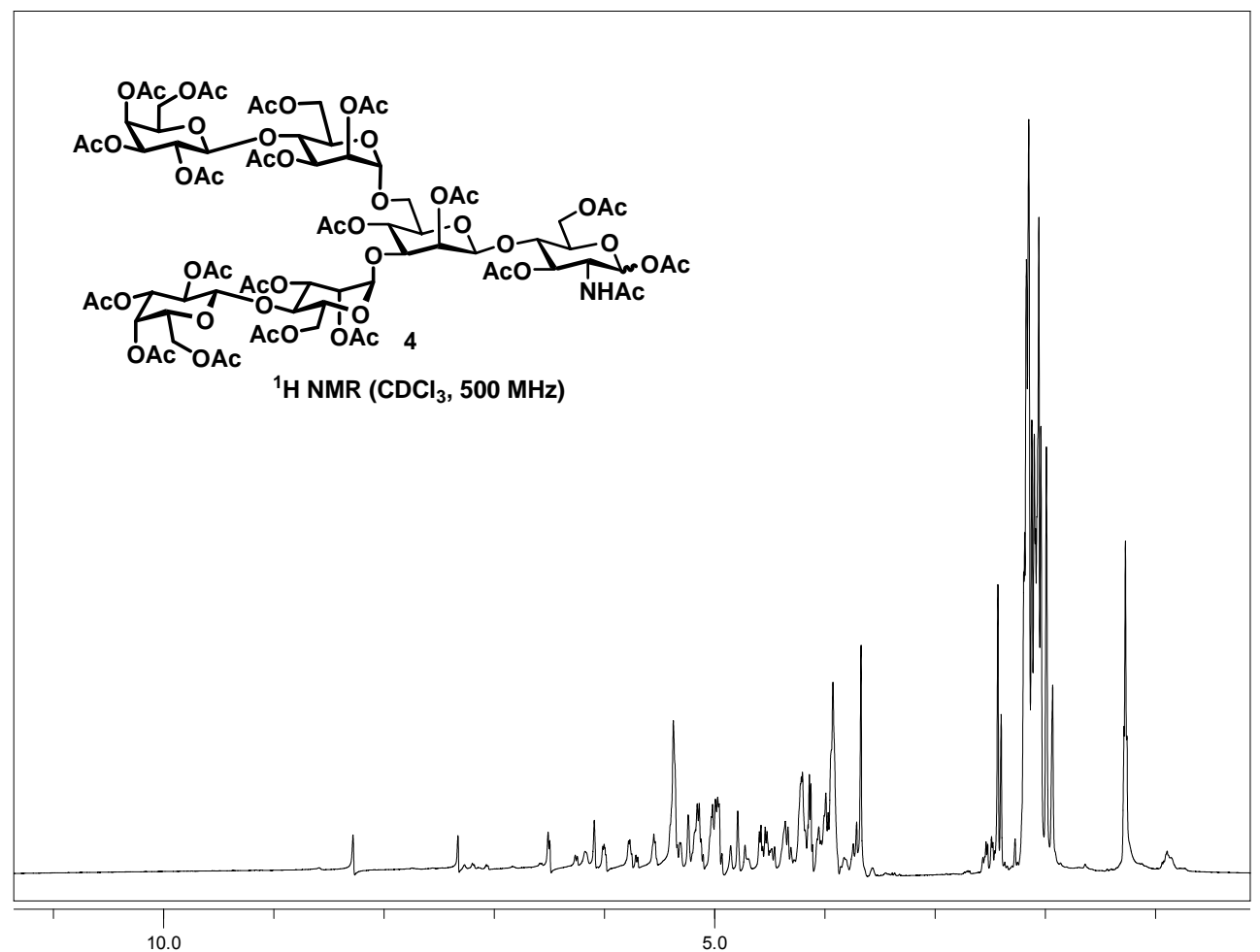

ppm (t1)

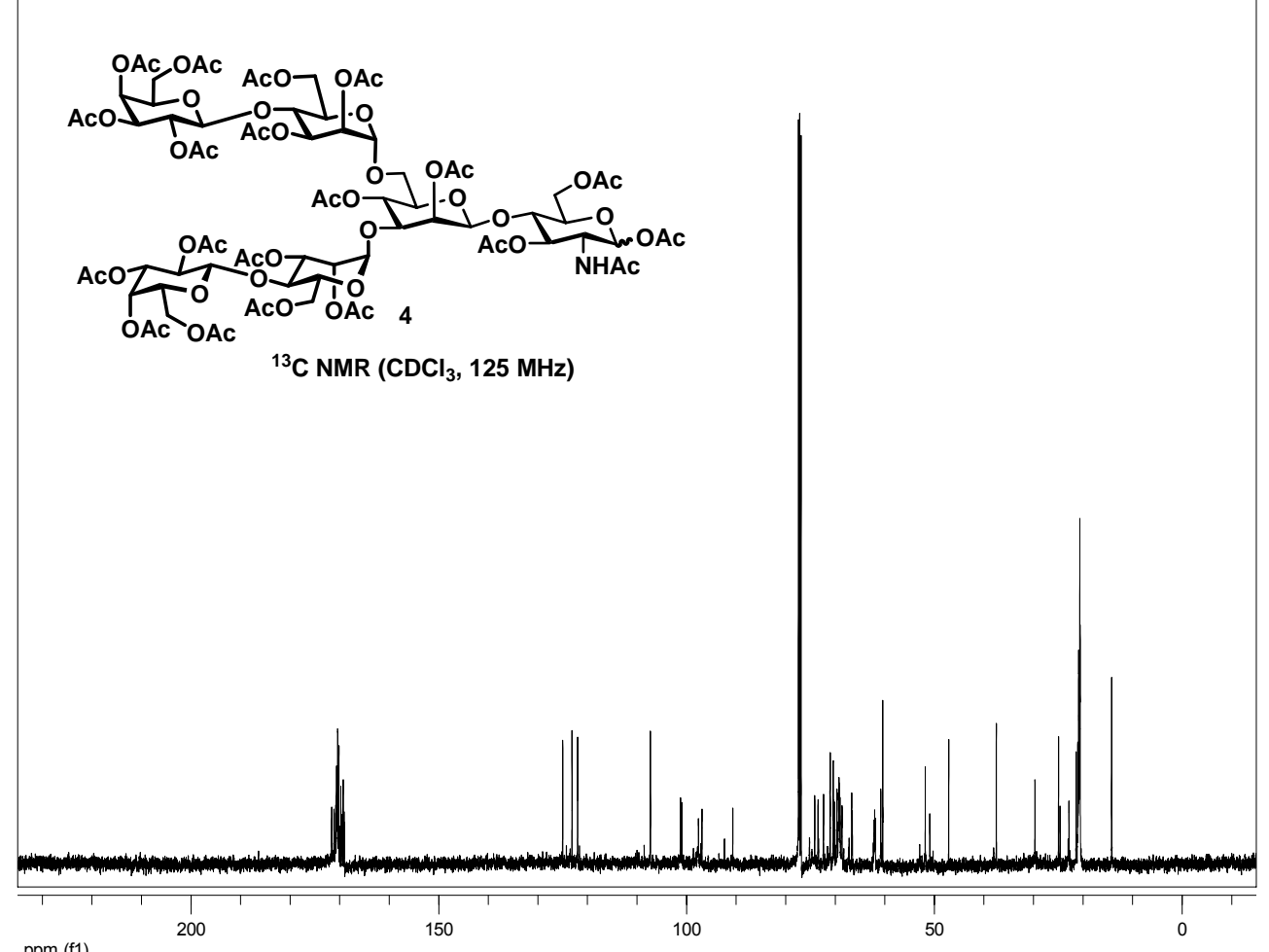

ppm (f1) 

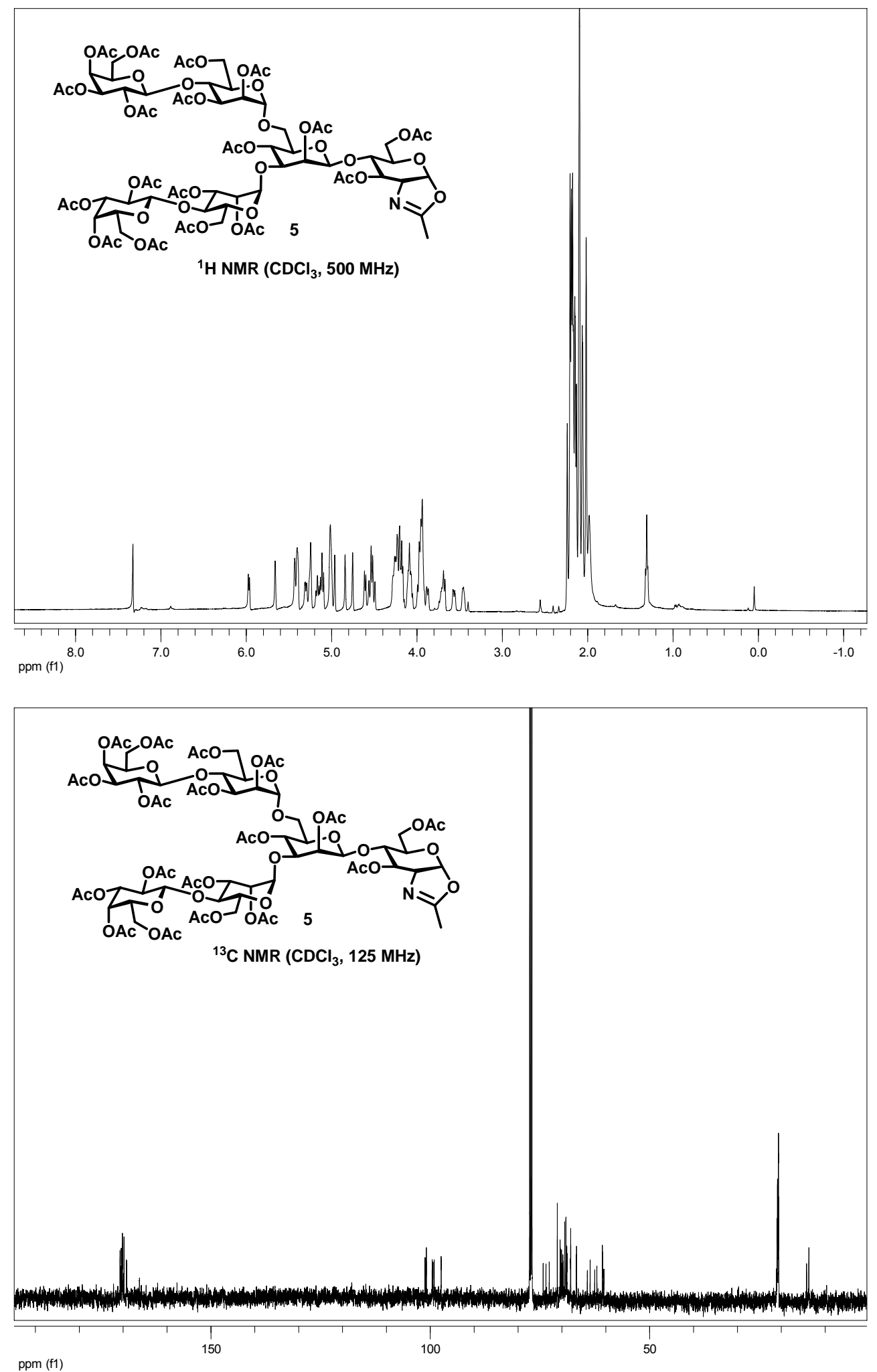

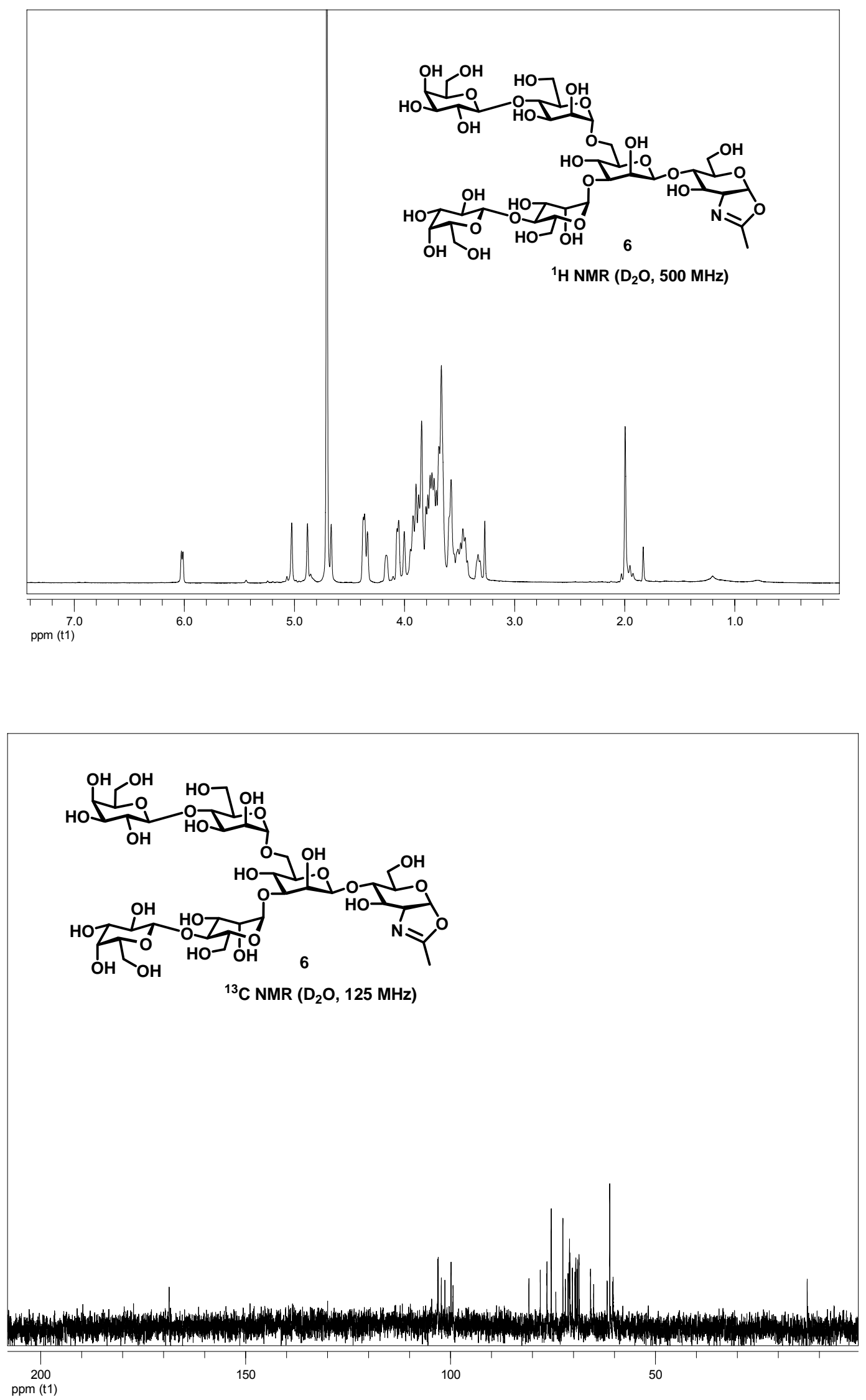

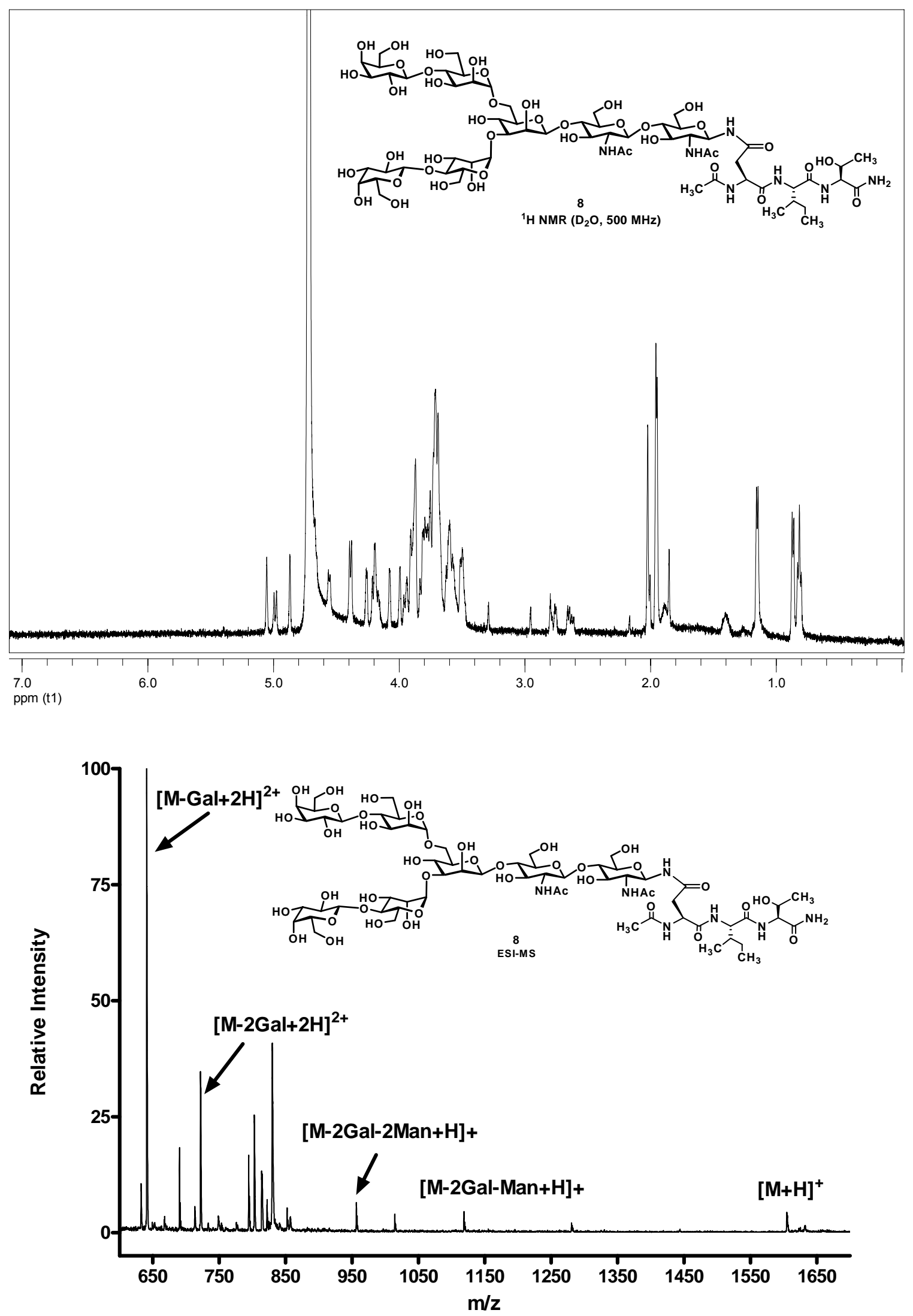